\title{
Early hypopharyngeal cancer treated with different therapeutic approaches: a single-institution cohort analysis
}

\author{
Nalee Kim, MD, Jeongshim Lee, MD, Kyung Hwan Kim, MD, Jong Won Park, MD, Chang Geol Lee, MD, PhD, \\ Ki Chang Keum, MD, PhD
}

Department of Radiation Oncology, Yonsei University College of Medicine, Seoul, Korea

\begin{abstract}
Purpose: Early hypopharyngeal squamous cell carcinoma (HPSCC) is a rarely diagnosed disease, for which the optimal treatment has not been defined yet. We assessed patterns of failure and outcomes in early HPSCC treated with various therapeutic approaches to identify its optimal treatment.

Materials and Methods: Thirty-six patients with stage I $(n=10)$ and II $(n=26)$ treated between January 1992 and March 2014 were reviewed. Patients received definitive radiotherapy (RT) (R group, $n=10)$, surgery only (S group, $n=19$ ), or postoperative RT (PORT group, $n=7$ ). All patients in both the $R$ and PORT groups received elective bilateral neck irradiation. In the $S$ group, 7 patients had ipsilateral and 8 had bilateral dissection, while 4 patients had no elective dissection.

Results: At a median follow-up of 48 months, the 5 -year locoregional control (LRC) rate was $65 \%$. Six patients had local failure, 1 regional failure (RF), 3 combined locoregional failures, and 2 distant failures. There was no difference in 5 -year LRC among the $R$, $S$, and PORT groups $(p=0.17)$. The presence with a pyriform sinus apex extension was a prognosticator related to $\operatorname{LRC}(p=0.01)$ in the multivariate analysis. Patients with a bilaterally treated neck showed a trend toward a lower RF rate $(p=0.08)$.

Conclusion: This study shows that patients with early stage HPSCC involving the pyriform sinus apex might need a tailored approach to improve LRC. Additionally, our study confirms elective neck treatment might have an efficacious role in regional control.
\end{abstract}

Keywords: Hypopharyngeal neoplasms, Treatment failure, Radiotherapy, Surgery

\section{Introduction}

Early-stage hypopharyngeal squamous cell carcinomas (HPSCCS) (stage I or II) have been reported in less than 20\% of patients with HPSCC due to advanced primary disease and extensive regional lymph node involvement before diagnosis because of the nonspecific nature of the early symptoms until extension to adjacent structures and abundant Iymphatic drainage of the hypopharynx $[1,2]$.
Because of the rarity of early HPSCC, few reports regarding treatment outcomes of early HPSCC have been published. In addition, owing to uncertainty about optimal treatment, a variety of treatment options have been considered for patients with early-stage HPSCC to preserve the larynx in clinical practice. Treatment options include endoscopic removal, open partial laryngopharyngectomy, total laryngectomy with partial pharyngectomy, and definitive radiotherapy (RT) [3]. National Comprehensive Cancer Network (NCCN) Guidelines [4] suggest

Received 16 March 2016, Revised 6 May 2016, Accepted 1 September 2016.

Correspondence: Ki Chang Keum, MD, PhD, Department of Radiation Oncology, Yonsei Cancer Center, Yonsei University College of Medicine, 50-1 Yonsei-ro, Seodaemun-gu, Seoul 03722, Korea. Tel: +82-2-2228-8112, Fax: +82-2-312-9033, E-mail: KCKEUM@yuhs.ac

(c) This is an Open Access article distributed under the terms of the Creative Commons Attribution Non-Commercial License (http://creativecommons.org/ licenses/by-nc/4.0/) which permits unrestricted non-commercial use, distribution, and reproduction in any medium, provided the original work is properly cited.

www.e-roj.org 
definitive RT or partial laryngopharyngectomy with ipsilateral or bilateral neck dissection for early-stage HPSCC. Factors that affect treatment options included the tumor extent, volume of the tumor, patient preference, patient age, comorbidity, physician experiences, and physician and institutional preferences.

At our institution, patients with early HPSCC are treated with either RT and surgery alone or a combined modality with surgery followed by postoperative RT. We assessed patterns of failure and outcomes in patients with early HPSCC who were treated with different therapeutic approaches in order to determine the optimal treatment strategy.

\section{Materials and Methods}

\section{Study population}

We identified 46 consecutive patients diagnosed at our institution between January 1992 and March 2014. Inclusion criteria were as follows: 1) patients with pathologically confirmed hypopharyngeal squamous cell carcinoma; 2) patients with newly diagnosed and previously untreated hypopharyngeal cancer; and 3) patients with T1/2N0M0 staging (American Joint Committee on Cancer [AJCC] staging). Three patients with synchronous malignancy, 2 with pathologically proven mucoepidermoid carcinoma, and 1 with myxoid liposarcoma were excluded. Four patients who underwent treatment at other institutions were excluded because there was limited follow-up information that could be used to assess the clinical outcomes. A total of 36 patients were analyzed in this retrospective study.

All patients underwent pretreatment evaluation, physical examination with laryngoscopy, and radiologic imaging such as computed tomography (CT) and magnetic resonance imaging (MRI) of the head and neck region. All patients were staged according to the 2010 AJCC TNM staging system (7th edition). There were 10 patients with stage I (tumor limited to one subsite of the hypopharynx and $2 \mathrm{~cm}$ or less in greatest dimension), and 26 with stage II (tumor invading more than one subsite of the hypopharynx or an adjacent site, or measuring more than $2 \mathrm{~cm}$ but not more than $4 \mathrm{~cm}$ in the greatest dimension without fixation of the hemilarynx). Patients treated with definitive RT were staged clinically based on physical examination and images, while those treated with surgery or surgery followed by postoperative RT were staged pathologically based on surgical pathologic specimens. The definition of the resection margin was based on pathologic reports. Achievement of less than $5 \mathrm{~mm}$ margins including tumor cells abutting the resection margins was defined as a close margin, and extension of tumor cells was defined as a positive margin. All other instances were defined as clear margins.

Among 36 patients, 10 were treated with definitive RT (R group), 19 with surgery only (S group), and 7 with surgery followed by postoperative RT (PORT group).

\section{Treatment}

Each treatment option was selected by the multidisciplinary head and neck cancer team according to age, medical comorbidities, and patients' and physicians' preferences. With the upfront approach, the location of the tumor was an important factor. For example, 2 patients with tumors located in the post-cricoid area, where difficult surgical techniques were required, were treated with definitive RT, and of the 14 patients with tumors in the posterior pharyngeal wall that were able to be resected relatively easily, all underwent surgery except for one patient who underwent definitive RT according to the patient's preference. Adjuvant treatments following surgery were determined based on pathologic reports. Positive or close resection margins were preferentially considered as determinants for adjuvant RT.

The $S$ group $(n=19)$ included mass excision $(n=2)$, partial pharyngectomy $(n=11)$, total laryngectomy with partial pharyngectomy $(n=4)$, and total pharyngolaryngectomy ( $=2$ ). In the $R$ group ( $n=10), 9$ patients underwent CT-based RT planning with either 3-dimensional conformal RT (3D-CRT, $n=3)$ or intensity-modulated RT (IMRT) ( $=6)$, and only one patient in the $\mathrm{R}$ group underwent 2D-based RT. The treatment plan with 2D-based RT encompassed the whole hypopharynx using two lateral beams for 70.2 Gy with a fraction dose of $1.8 \mathrm{~Gy}$ and covered the bilateral neck level II to SCL for $45 \mathrm{~Gy}$ with a fraction dose of $1.8 \mathrm{~Gy}$. For all other patients treated with 3D-CRT or IMRT, the gross tumor volume (GTV) at the primary site plus generally $3 \mathrm{~mm}$ margins was defined as the planning target volume of the primary site (PTVp), which was treated with a median total dose of $68.4 \mathrm{~Gy}$ (range, 60.8 to $70.2 \mathrm{~Gy}$ ) and a median fraction dose of $2.1 \mathrm{~Gy}$ (range 1.8 to 2.3 Gy). For all patients in the $\mathrm{R}$ group, the clinical target volume of the neck node area (CTVn) was defined as the bilateral neck level II to IV, and in 2 patient, the bilateral retropharyngeal nodal regions were added to the CTVn. After delineating CTVn, the additional $3 \mathrm{~mm}$ margins were added to planning target volume of the neck node area (PTVn) (Fig. 1 ). A median total dose of $54 \mathrm{~Gy}$ (range, 30.6 to $55.8 \mathrm{~Gy}$ ) with daily fraction doses of 1.8 Gy was prescribed for the PTVn. For the PORT group (n 


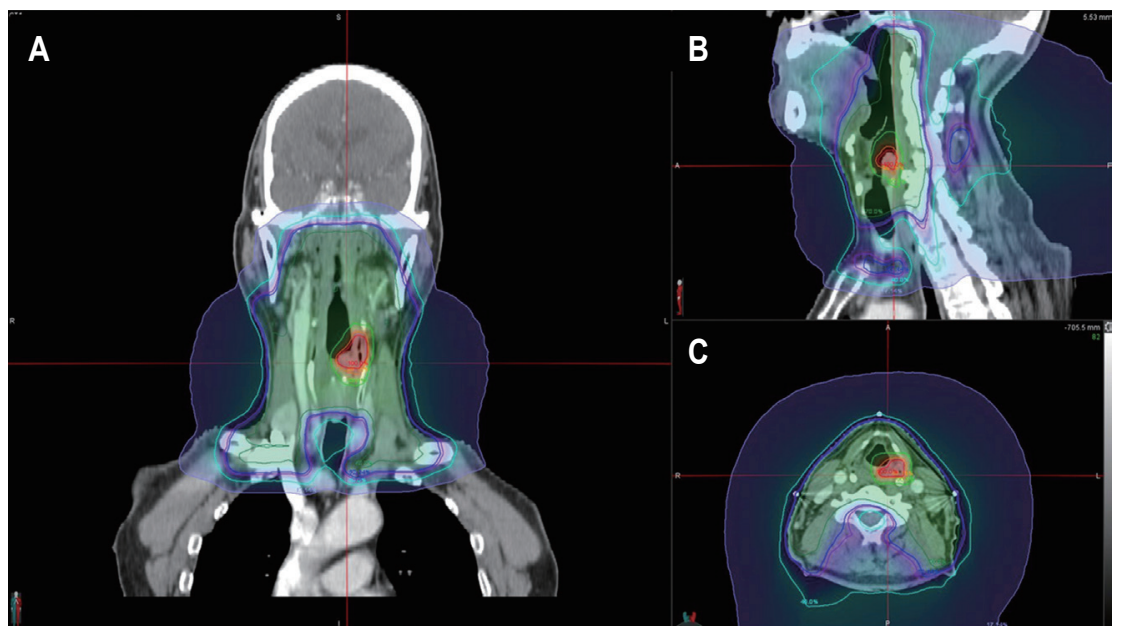

Fig. 1. Computed tomography images showing an example of radiotherapy planning with intensity-modulated radiotherapy. A 72-year-old male patient was diagnosed with early hypopharyngeal squamous cell carcinoma involving the left aryepiglottic fold without pyriform sinus apex extension. The planning target volume for gross tumor (PTVp) covered primary site with margins. The planning target volume for elective neck node area (PTVn) covered bilateral neck level II to IV and retropharyngeal nodal regions with additional margins. The prescribed doses to PTVp and PTVn were 66 Gy in 2.2 Gy and $45 \mathrm{~Gy}$ in $1.8 \mathrm{~Gy}$, respectively.

= 7), 6 patients underwent partial pharyngectomy and one patient underwent mass excision. Six patients underwent CTbased RT planning with either 3D-CRT $(n=5)$ or IMRT $(n=1)$, and 1 patient underwent 2D-based RT. 2D-based RT planning covered the postoperative tumor bed with a total dose of 54 Gy and a daily fraction dose of $1.8 \mathrm{~Gy}$ and the upper neck levels II to III were treated with a total dose of $45 \mathrm{~Gy}$ and a daily fraction dose of $1.8 \mathrm{~Gy}$. In 6 patients with CT-based RT planning, the PTVp was defined as the postoperative tumor bed plus $3 \mathrm{~mm}$ margins and was prescribed a median total dose of $63 \mathrm{~Gy}$ (range, 59.4 to $66.6 \mathrm{~Gy}$ ) with a daily fraction dose of $1.8 \mathrm{~Gy}$ (range, 1.8 to $2.0 \mathrm{~Gy}$ ). The PTVn was defined as the bilateral neck levels II to IV, and in 1 patient, the bilateral retropharyngeal nodal regions were added to the PTVn. A median total dose of $45 \mathrm{~Gy}$ (range, 41.4 to $54 \mathrm{~Gy}$ ) with a daily fraction doses of $1.8 \mathrm{~Gy}$ (range, 1.8 to $2.0 \mathrm{~Gy}$ ) was prescribed for the PTVn.

Treatment-related toxicities were assessed using the Common Terminology Criteria for Adverse Events (CTCAE, ver. 4.03) based on limited medical records. Treatment toxicities were not fully accessible to the entire cohort, particularly for the $S$ group, for which there was limited information. Only 8 patients in the $S$ group were recorded as having treatment toxicities. Information on acute toxicities related to RT was available for 17 patients of the $R$ and PORT groups. Late toxicities were assessed in only 11 patients.

\section{Statistical analysis}

The primary endpoint of this study was the locoregional control rate $(\mathrm{LRC})$, which is defined as complete and persistent regression of the tumor in the primary site or regional lymph nodes. The LRC was calculated from the start of treatment to the date of locoregional failure diagnosis or date of last follow-up. The Kaplan-Meier method was used to estimate the $L R C$ rate. The survival curves were compared using the log-rank test. To determine the effects of distinct prognostic factors on survival, multivariate analysis was performed using a Cox's regression model. Differences in patient characteristics of the pyriform sinus (PS) extension and elective neck treatment were assessed using the Pearson $\chi^{2}$-test. Differences in treatment options were assessed using the Fisher exact test. In all statistical analyses, $p<0.05$ was considered to be significant. Statistical analysis was carried out using IBM SPSS ver. 20 (IBM, Armonk, NY, USA).

\section{Results}

\section{Patient and tumor characteristics}

The median follow-up period for the entire cohort was 48 months (range, 5 to 164 months). Median age at diagnosis was 65 years (range, 46 to 85 years) and most patients were men (91.7\%). Twenty-seven patients (75\%) were ex-smokers with a median of 30 pack-years. The primary site of the tumor was the PS in 20 patients (55.6\%), posterior pharyngeal wall in 14 patients (38.9\%), and the post cricoid area in 2 patients (5.6\%). The PS apex extension was identified through a laryngoscope in 14 patients (38.9\%). The median tumor size was $2.3 \mathrm{~cm}$ (range, 0.5 to $4.0 \mathrm{~cm}$ ). There was no statistically significant difference in patient and tumor characteristics among the treatment groups. The baseline patient and tumor characteristics are shown in Table 1.

\section{Treatment characteristics}

In the S group, open surgery was performed in 16 patients 
Table 1. Patient characteristics

\begin{tabular}{|c|c|c|c|c|c|}
\hline Characteristic & Total $(n=36)$ & $R$ group $(n=10)$ & S group $(n=19)$ & PORT group $(n=7)$ & $p$-value \\
\hline Age (yr) & $65(46-85)$ & $69(46-83)$ & $65(53-85)$ & $61(49-80)$ & 0.78 \\
\hline$<65$ & $17(47.2)$ & $4(40.0)$ & $9(47.4)$ & $4(57.1)$ & \\
\hline$\geq 65$ & $19(52.8)$ & $6(60.0)$ & $10(52.6)$ & $3(42.9)$ & \\
\hline Sex & & & & & 0.67 \\
\hline Male & 33 (91.7) & $9(90.0)$ & 17 (89.5) & $7(100)$ & \\
\hline Female & $3(8.3)$ & $1(10.0)$ & $2(10.5)$ & $0(0)$ & \\
\hline Smoking history & & & & & 0.68 \\
\hline None & 7 (19.4) & $2(20.0)$ & $3(15.8)$ & $2(28.6)$ & \\
\hline Ex-smoker & $27(75)$ & $8(80.0)$ & $15(78.9)$ & $4(57.1)$ & \\
\hline Unknown & $2(5.6)$ & $0(0)$ & $1(5.3)$ & $1(14.3)$ & \\
\hline Pack-year & $30(0-90)$ & $28(0-70)$ & $30(0-80)$ & $20(0-90)$ & \\
\hline Alcohol history & & & & & 0.41 \\
\hline None & $5(13.9)$ & $0(0)$ & $3(15.8)$ & $2(28.6)$ & \\
\hline Social & $23(63.9)$ & $8(80.0)$ & $10(52.6)$ & $5(71.4)$ & \\
\hline Heavy & 7 (19.4) & $2(20.0)$ & $5(26.3)$ & $0(0)$ & \\
\hline Unknown & $1(2.8)$ & $0(0)$ & $1(5.3)$ & $0(0)$ & \\
\hline ECOG performance status & & & & & 0.39 \\
\hline $0-1$ & $34(94.4)$ & $10(100)$ & $17(89.5)$ & $7(100)$ & \\
\hline $2-4$ & $2(5.6)$ & $0(0)$ & $2(10.5)$ & $0(0)$ & \\
\hline Subsite & & & & & 0.08 \\
\hline PS & $20(55.6)$ & $7(70.0)$ & $10(52.6)$ & $3(42.9)$ & \\
\hline PPW & $14(38.9)$ & $1(10.0)$ & $9(47.4)$ & $4(57.1)$ & \\
\hline PC & $2(5.6)$ & $2(20.0)$ & $0(0)$ & $0(0)$ & \\
\hline T stage & & & & & 0.67 \\
\hline $\mathrm{T} 1$ & $10(27.8)$ & $3(30.0)$ & $6(31.6)$ & $1(14.3)$ & \\
\hline $\mathrm{T} 2$ & $26(72.2)$ & $7(70.0)$ & $13(68.4)$ & $6(85.7)$ & \\
\hline Median volume (mL) & $6.6(2.6-12.8)$ & $6.4(3.6-8.21)$ & $6.2(2.6-12.8)$ & $6.4(3.6-8.2)$ & \\
\hline PS apex & & & & & 0.28 \\
\hline Free & $22(61.1)$ & $8(80.0)$ & $11(57.9)$ & $3(42.9)$ & \\
\hline Extension & $14(38.9)$ & $2(20.0)$ & $8(42.1)$ & $4(57.1)$ & \\
\hline Tumor differentiation & & & & & 0.48 \\
\hline WD/MD & $33(91.7)$ & $8(80.0)$ & $18(94.7)$ & $7(100)$ & \\
\hline PD & $3(8.3)$ & $2(20.0)$ & $1(5.3)$ & $0(0)$ & \\
\hline Resection margin & & & & & 0.03 \\
\hline Clear & - & - & $13(68.4)$ & $1(14.3)$ & \\
\hline Close/positive & - & - & $6(31.6)$ & $6(85.7)$ & \\
\hline
\end{tabular}

Values are presented as median (range) or number (\%).

$\mathrm{R}$, definitive radiotherapy; S, surgery; PORT, surgery followed by adjuvant radiotherapy; ECOG, Eastern Cooperative Oncology Group; PS, pyriform sinus; PPW, posterior pharyngeal wall; PC, post cricoid area; WD, well-differentiated; MD, moderate-differentiated; PD, poorly differentiated.

(84.2\%) and laser or trans-oral surgery (TORS) in 3 patients (15.8\%). In the PORT group, 3 patients (42.9\%) underwent open surgery, and 4 (57.1\%), TORS or laser surgery. There was a trend toward higher prevalence of minimally invasive surgery such as laser or TORS in the PORT group than in the S group $(p=0.06)$. The resection margin $(R M)$ status was statistically different between both groups that underwent surgery ( $S$ group and PORT group) ( $p=0.03)$. A close or positive RM was observed in 6 patients (31.6\%) in the $S$ group and 6 (84.7\%) in the PORT group.

All patients in the $R$ group underwent bilateral elective neck treatment. In the S group, no elective neck treatment was performed in 4 patients (21.1\%); ipsilateral neck dissection was performed in 7 patients (36.8\%), and bilateral neck dissection in 8 patients (42.1\%). In the PORT group, ipsilateral elective neck dissection was performed in 3 patients (42.9\%), and bilateral neck dissection in 3 patients (42.9\%); no elective neck dissection was performed in 1 patient. Patients in the PORT 


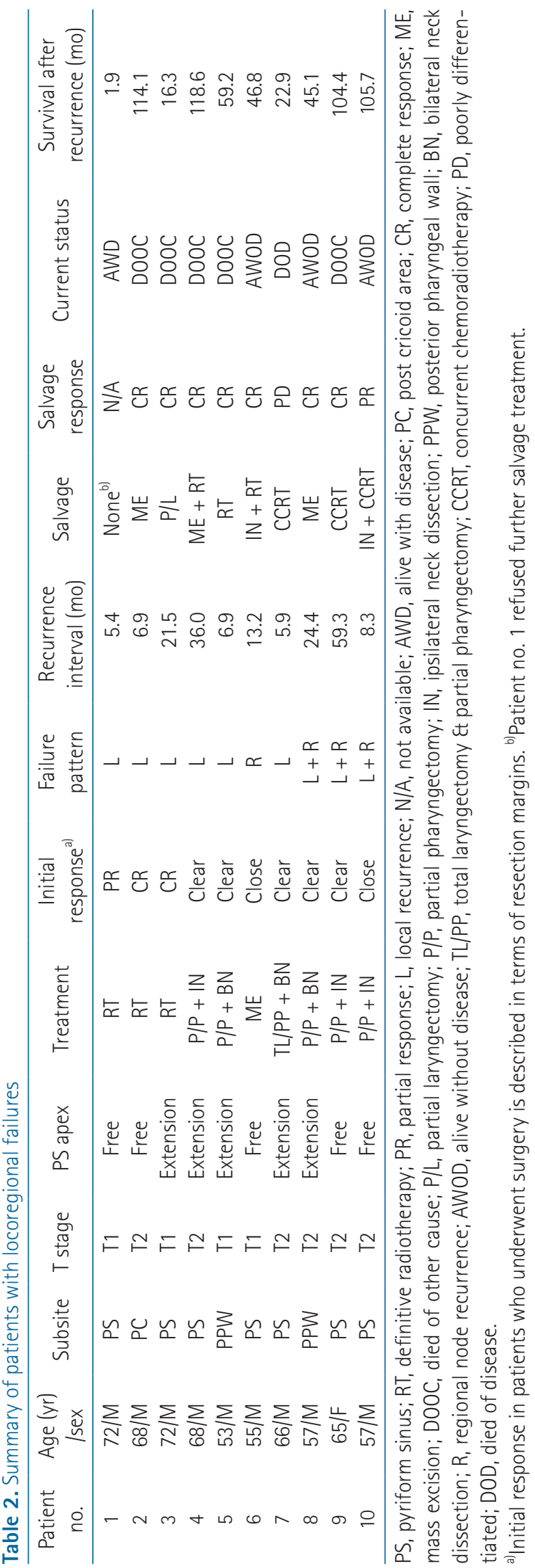

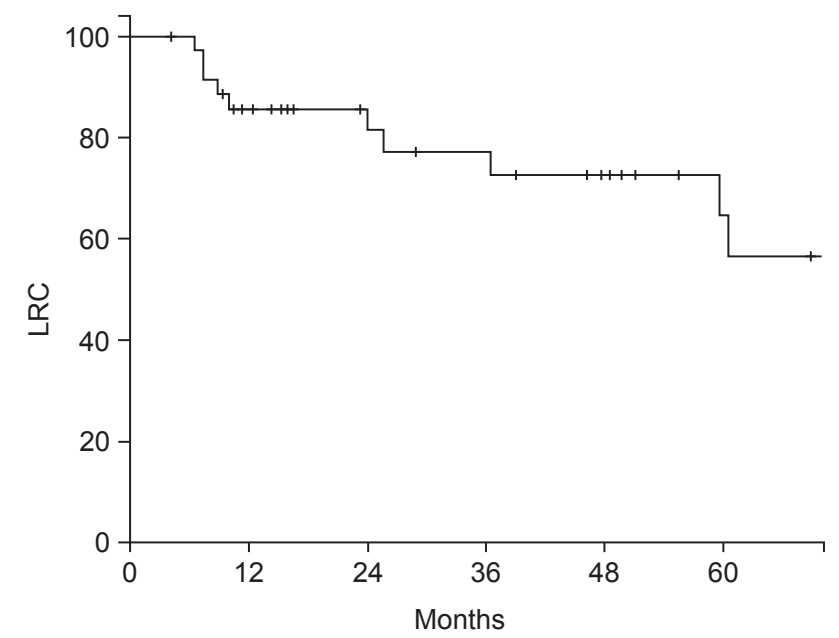

Fig. 2. Locoregional control rate (LRC) for the entire cohort.

group were treated with elective irradiation to bilateral neck nodes.

\section{Patterns of failure}

With a median time to recurrence of 10.5 months (range, 5.9 to 59.3 months), among all patients, 12 patients (33.3\%) developed failures, including 6 with local failure (LF), 1 with regional failure (RF), 3 with combined loco-regional failures (LRFs), and 2 with distant metastases (DM). The PORT group did not have any treatment failures but the $\mathrm{R}$ group experienced failures in 4 cases, of which 3 were LF and 1 was DM. The $S$ group had 8 treatment failures (42.1\%) including 3 LF, 1 RF, 3 combined LRF, and $1 \mathrm{DM}$.

In patients with $L F, R F$ and $L R F$, salvage treatment and outcomes are summarized in Table 2. Except for 1 patient who refused, 9 patients underwent salvage treatment. Of these patients, 3 underwent salvage surgery alone; a supraglottic partial laryngectomy was performed in 1 patient, and mass excision in 2 patients. Salvage RT was performed in 1 patient and concurrent chemoradiotherapy (CRT) in 2 patients. Three patients underwent salvage surgery followed by adjuvant treatment; 2 patients had a neck dissection with postoperative RT; and 1 patient had mass excision followed by neck node RT. Except for 1 patient with primary tumor bed and retropharyngeal node recurrence, 8 patients showed a complete response after salvage treatment. The 5 -year ultimate $L C$ after salvage treatment was $79.8 \%$.

\section{Locoregional/regional control and prognostic factors}

The overall 5-year LRC was $64.6 \%$ (Fig. 2). There was no difference in the 5 -year LRC among the 3 groups $(R, S$, 
A

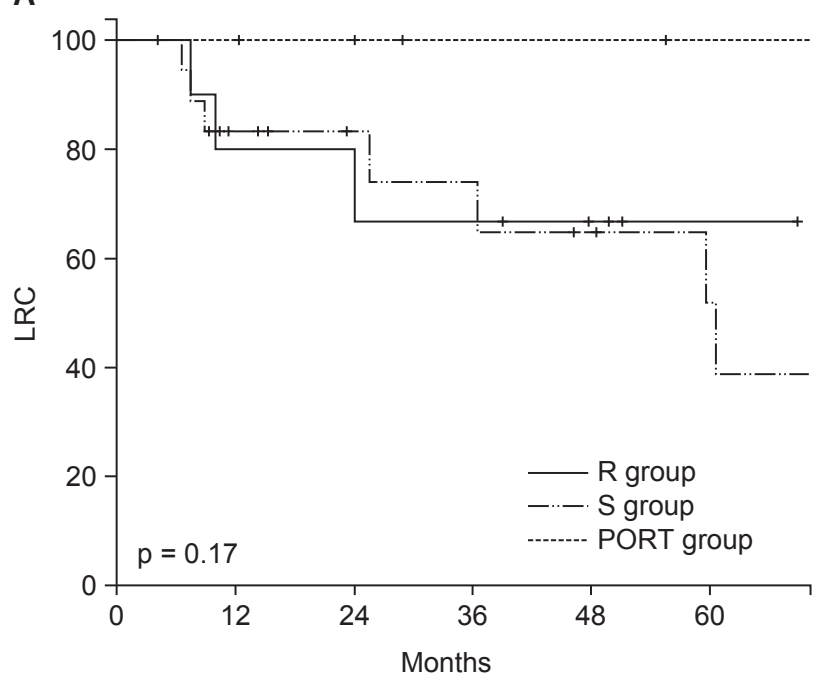

B

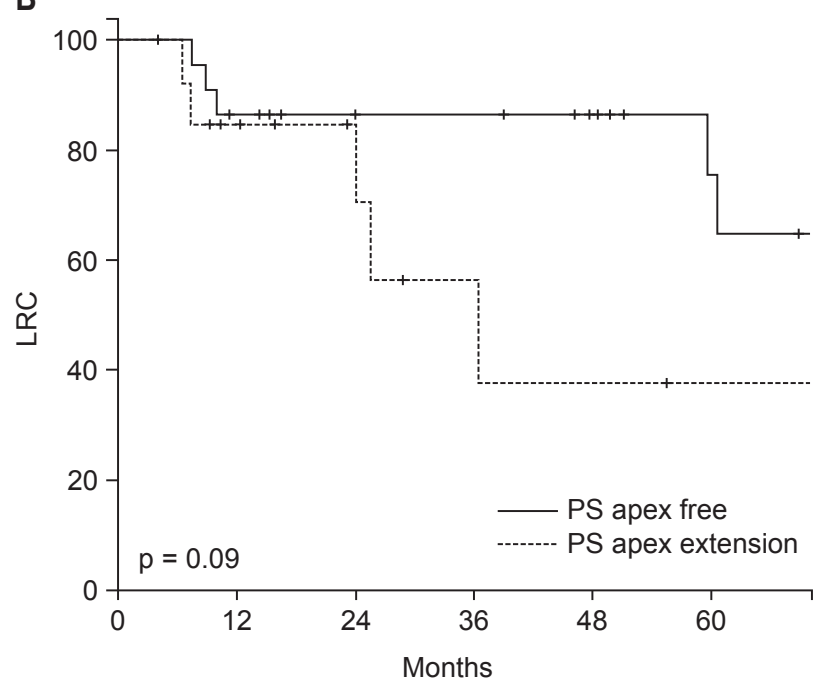

Fig. 3. Locoregional control rate (LRC) according to the treatment approach (A) and the pyriform sinus (PS) apex extension (B). $R$, definitive radiotherapy; $S$, surgery only; PORT, surgery followed by adjuvant radiotherapy.

Table 3. Univariate and multivariate analysis for association of factors with LRC

\begin{tabular}{|c|c|c|c|c|c|}
\hline & \multicolumn{2}{|c|}{ Univariate analysis } & \multicolumn{3}{|c|}{ Multivariate analysis } \\
\hline & 5-yr LRC (\%) & $p$-value & $\mathrm{HR}$ & $95 \% \mathrm{Cl}$ & p-value \\
\hline Age (yr) & & 0.41 & & & - \\
\hline$<65$ & 80.2 & & - & - & \\
\hline$\geq 65$ & 49.0 & & - & - & \\
\hline Subsite & & 0.24 & & & - \\
\hline PS & 57.1 & & - & - & \\
\hline PPW & 79.1 & & - & - & \\
\hline PC & 50.0 & & - & - & \\
\hline T stage & & 0.14 & & & 0.14 \\
\hline $\mathrm{T} 1$ & 60.0 & & Ref. & - & \\
\hline $\mathrm{T} 2$ & 66.9 & & 0.36 & $0.09-1.40$ & \\
\hline PS apex & & 0.09 & & & 0.01 \\
\hline Free & 75.6 & & Ref. & - & \\
\hline Extension & 37.6 & & 7.10 & $1.50-33.61$ & \\
\hline Tumor differentiation & & 0.29 & & & - \\
\hline WD/MD & 61.4 & & - & - & \\
\hline PD & 100 & & - & - & \\
\hline Treatment option & & 0.17 & & & \\
\hline S group & 51.9 & & Ref. & - & - \\
\hline$R$ group & 66.7 & & 0.68 & $0.12-3.76$ & 0.66 \\
\hline PORT group & 100 & & 0.00 & $0.00-\mathrm{NA}$ & 0.97 \\
\hline Neck treatment & & 0.68 & & & \\
\hline None/ipsilateral & 56.8 & & Ref. & - & - \\
\hline Bilateral & 71.8 & & 1.67 & $0.33-8.30$ & 0.54 \\
\hline
\end{tabular}

LRC, locoregional control rate; HR, hazard ratio; $\mathrm{Cl}$, confidence interval; PS, pyriform sinus; PPW, posterior pharyngeal wall; PC, post cricoid area; $W D$, well-differentiated; $M D$, moderate-differentiated; $P D$, poorly differentiated; $S$, surgery only; $R$, definitive radiotherapy; PORT, surgery followed by adjuvant radiotherapy; NA, not available. 

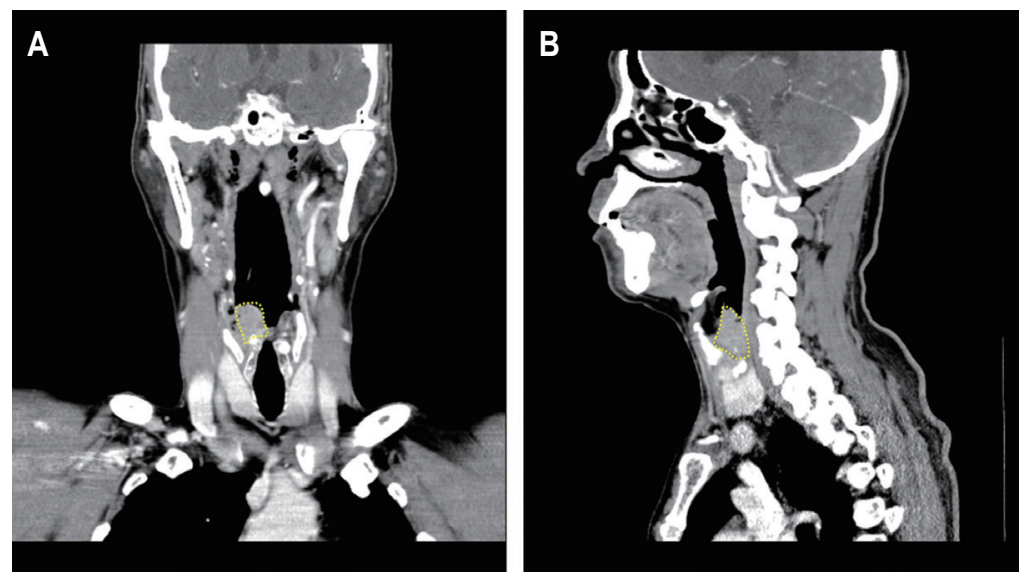

Fig. 4. Computed tomography images showing an example of hypopharyngeal squamous cell carcinoma (HPSCC) with a pyriform sinus (PS) apex extension (tumor mass was marked by a dashed line). A 66-year-old male patient was diagnosed with early HPSCC involving right PS and right aryepiglottic fold. The patient showed a clear resection margin after total laryngectomy and partial pharyngectomy with bilateral neck dissection. After 6 months, local failure at the primary tumor bed developed. He underwent concurrent chemoradiotherapy. At 23 months after the local failure, hematogenous lung metastasis developed, and the patient died of disease.

and PORT 66.7\%, 51.9\%, and 100\%; $p=0.17$ ) (Fig. 3A) Furthermore, there was no statistically significant predictive factor of LRC based on the univariate analysis (Table 3). The PS apex extension showed a borderline significance related to 5 -year LRC in the univariate analysis (75.6\% vs. 37.6\%; $p=$ 0.09) (Fig. 3B). When performing a multivariate analysis using factors with a $p$-value of $<0.20$ on univariate analysis, the LRF in patients with a PS apex extension was significantly higher (hazard ratio $[\mathrm{HR}], 7.10 ; 95 \%$ confidence interval $[\mathrm{Cl}], 1.50$ to 33.61; $p=0.01$ ) (Table 3, Fig. 4) than that in patients with an intact PS apex considering the T stage, treatment modality, and elective neck node treatment.

Patients with a bilaterally treated neck had a lower RF rate compared to untreated or ipsilateral treated patients (4\% vs. $27 \% ; p=0.08$ ). Of the 4 nodal recurrent events, 1 nodal failure was observed in a patient initially treated with bilateral neck dissection, and 3 in patients with initially untreated neck nodes; 1 patient was initially treated without neck dissection, and 2 patients with contralateral neck node failures were initially treated with ipsilateral neck node dissection.

\section{Toxicity}

Fourteen patients (38.9\%) experienced more than grade 3 treatment related toxicities. There was no statistically significant difference in the proportion of patients who experienced grade 3 toxicities ( $p=0.72)$, which included 3 patients in the $\mathrm{R}$ group, 8 patients in the $\mathrm{S}$ group, and 3 patients in the PORT group.

Acute toxicities of grade 3 or higher including wound dehiscence and fistula formation after surgery were observed in 6 patients of the S group and in 3 patients of the PORT group. All patients who underwent RT ( $R$ or PORT group) experienced acute grade 1 or 2 oral mucositis; however, all patients recovered within 1 month after RT. One patient in the $\mathrm{R}$ group underwent both tracheostomy and gastrostomy due to grade 4 pharyngitis and esophagitis. Scheduled RT was interrupted due to grade 3 generalized weakness in another patient in the R group who finished RT early after 27 treatment sessions. Late toxicities of grade 3 or higher stenosis or pharyngitis was observed in 11 patients; 2 patients in the $\mathrm{R}$ group, 4 patients in the $S$ group, and 2 patients in the PORT group (20.0\% vs. $28.6 \%$ vs. $21.1 \% ; p=1.00)$. These patients underwent delayed gastrostomy or tracheostomy.

\section{Discussion and Conclusion}

In this study, we assessed LRC and patterns of treatment failure in patients with early-stage HPSCC treated with various therapeutic approaches. The previous studies reported that the LRC rate of early-stage HPSCC was $60 \%$ to $88 \%$ when treated either by surgery [5-7] or by definitive RT [8-13]. We found that the 5-year LRC rate of these patients was 65\%, which was comparable to other studies. We also analyzed patterns of $L R F$, including 6 cases of $L F, 1$ of $R F$, and 3 of combined LRF. Nakamura et al. [8] evaluated 115 patients with stage I-II HPSCC treated with RT with or without chemotherapy. This multi-institutional study reported that the $L R C$ rate was $78 \%$, with 25 cases of $L F, 9$ of RF, and 5 of combined failures. Sato et al. [12] reported a 56\% 5-year LRC rate in 33 stage I-II HPSCC patients treated with either RT or CCRT. Among the 33 patients, LRF occurred in 13 patients: 8 had LF, 1 had RF, and 3 had combined failures. Kuo et al. [7] reported 53 patients treated with either open partial laryngopharyngectomy or endoscopic laser microsurgery had a 5-year LRC of 71\%. Of the 53 patients, 13 experienced failures (8 LF and 5 RF).

Furthermore, the 5 -year LRC rate in early-stage HPSCC 
with PS apex extension was $37.6 \%$ compared to $75.6 \%$ in tumors without apex extension $(p=0.09)$. However, it should be interpreted cautiously in terms of both a small number of patients and borderline significance. There was no difference in $L R C$ rate among treatment groups whether with PS apex extension or not. In patients with PS apex extension, LRF occurred in 5 patients: 4 in the $S$ group (50\%), 1 in the R group (50\%). In patients without PS apex extension, 3 patients in the $S$ group (27\%) and 2 patients in the $R$ group (25\%) experienced LRF and there was no statistically significant difference.

We also found that PS apex extension was a significant prognosticator of LRC in multivariate analyses. Several previous reports have confirmed the correlation between PS apex extension and LRC [13-15]. Rabbani et al. [13] reported that borderline significance of PS apex involvement correlated to local control (79\% vs. $90 \%, p=0.11)$ in 84 HPSCC patients. Pameijer et al. [14] reported that the bulky apex disease significantly influenced the local control rate $(p=0.03)$ in HPSCC treated with definitive RT. Amdur et al. [15] reported on the treatment outcomes of 101 HPSCC patients with T1 $(n=22)$ and T2 $(n=79)$ cancer who were treated with RT with/without planned neck dissection. Patients with T1 HPSCC involving the PS apex showed significantly reduced LC compared to those without PS apex extension (33\% vs. 100\%; $p=0.02)$.

In the current study, 85\% (12 of 14) patients with a PS apex extension underwent surgery. Two patients with positive surgical margin who underwent minimally invasive surgery were treated with adjuvant RT, and did not show LRF during follow-up periods of 30 and 13 months, respectively. Additionally, two other patients with positive margins who underwent adjuvant RT lived without disease until the last follow-up (132.7 and 55.5 months, respectively). Conversely, 4 of $8(50 \%)$ patients with a PS extension who underwent surgery alone experienced LRF, even in case of negative surgical margins. This implies that appropriate adjuvant RT encompassing the primary tumor bed and elective neck lymph nodes should be considered in early HPSCC patients with PS apex extension. Especially, adjuvant RT could play a critical role in patients with preoperatively diagnosed PS apex extension because the probability of positive surgical margins increases in the era of minimally invasive surgery.

There was no RF in patients of R or PORT groups who received $R T$ covering the bilateral neck nodal chain, and only one case $(12.5 \%)$ of RF in the $\mathrm{S}$ group underwent bilaterally neck dissection. A favorable 5 -year LRC was shown in our study, although there was not a statistically significant difference in the bilaterally treated neck group compared to the ipsilateral or untreated neck group (56.8\% vs. $71.8 \%$; $p=0.68$ ), with no increase in rates of late toxicity. In this study, it was presumed that RT could play a role in elective neck node treatment to manage microscopic Iymphatic spread in patients treated with or without surgery. Owing to the rich lymphatic drainage of the hypopharyngeal region, occult nodal metastases including micro-metastases and contralateral neck metastasis were observed to range from $30 \%$ to $40 \%$ in clinically NO HPSCC $[16,17]$. For these patients, micro-metastases cannot be neglected due to the abundant lymphatic drainage; thus, omission of neck treatment leaves the probability of occult nodal failure [18], which correlates with poor LRC and ultimately poor distant control and overall survival [19]. Yoshimura et al. [20] showed the importance of locoregional field RT by reporting 77 patients with stage I or II hypopharyngeal cancer (HPC) treated with definitive RT either with or without chemotherapy. In this study, 66 of 77 patients (85\%) were treated with locoregional RT that electively encompassed the bilateral neck and nodal regions. Patients treated with a locoregional field had a statistically significant better 5 -year LC with laryngeal voice preservation in $73 \%$ compared to $42 \%$ among patients treated with a local field. Though locoregional RT could achieve a favorable outcome, treatment-related toxicities such as xerostomia should be considered due to the extent of the irradiated field. Comparable outcomes with less toxicity are expected in those treated in the era of IMRT $[21,22]$. In a meta-analysis of 871 patients treated with 2D-based RT or 3D-CRT versus IMRT [21], IMRT was found to reduce the incidence of grade 2-4 xerostomia ( $\mathrm{HR}, 0.76 ; 95 \% \mathrm{Cl}, 0.66$ to $0.87 ; \mathrm{p}<0.01)$ without compromising LRC or OS. A randomized study [22] also showed that IMRT significantly reduced the incidence and severity of xerostomia. Therefore, RT with advanced IMRT could be a tolerable treatment option in early HPC resulting in adequate coverage of bilateral neck node with reduced toxicities.

However, there is a still an issue of the extent of elective neck node treatment [23]. We observed 2 patients with contralateral RF, which accounted for $25 \%$ of those initially treated with ipsilateral neck dissection in the $S$ group. The current surgical approaches recommend selective neck dissection of cervical lymph node level II to IV to prevent occult nodal failure [24]. A few studies have recommended elective contralateral neck treatment for high-risk patients, namely those with tumor involving both the medial wall of the PS $[25,26]$ and the posterior pharyngeal wall [27]. Future 
research will be necessary to identify the prognostic factor(s) related to regional control in terms of optimal neck treatment.

In general, adjuvant RT improved LRC after surgery with adverse pathologic features, especially those involving RM [28]. Of the 12 patients with close or positive resection margins, 6 patients underwent adjuvant RT and showed no failure. Thus it can be assumed that adjuvant RT can prevent upcoming failures in patients with unfavorable postoperative pathologic features, although there was no significant statistical difference in failure. The addition of adjuvant RT after surgery achieved an excellent $L R C$ rate. Interestingly, although the PORT group had more adverse surgical RMs than the $S$ group (84.7\% vs. 31.6\%; $p=0.03$ ), there was no LF, RF and LRF in the PORT group compared to the $S$ group. In addition, while 1 patient (16.7\%) out of 6 patients with total laryngectomy or total pharyngolaryngectomy had positive margins, 11 patients (55.0\%) out of 20 patients with partial pharyngectomy or mass excision had close/positive margins. Organ conservation surgery, such as partial pharyngectomy and minimally invasive approaches, were likely to result in close or positive RMs due to the high predilection of submucosal tumor spread of HPSCC. Regarding the current trend with declining use of open or extensive surgery [29], appropriate adjuvant RT should be considered with elective neck node irradiation to yield a good LRC.

The findings of this study should be interpreted with an understanding of the following limitations. Due to the Iow prevalence of early HPSCC, the data of this study was comprised of a relatively small number of patients. The small sample size might have limited the possibility to detect statistically meaningful differences. Since our study did not include pathologically proven node positive patients in clinically node negative patients after surgery, it is difficult to compare the treatment outcomes directly between $\mathrm{R}$ and $\mathrm{S}$ or PORT. Therefore, we were not able to suggest statistically proven better treatment modality among $S, R$, and PORT. Despite the low number of patients, follow-up time for each patient was adequate to assess LRC according to each treatment. It is possible that the differences in the treatment approach over the periods of the study, such as a surgical approach or radiotherapy treatment technique (3D-CRT, IMRT), could result in a misinterpretation. Further investigation with large cohort is needed to evaluate the appropriate treatment option in early stage HPSCC. It should also be noted that clinical and functional data for treatment-related toxicities were not available. This could be possible source of underestimating toxicities.
In conclusion, we found that early stage HPSCC could achieve a favorable LRC by each treatment modality with optimized management of the elective neck nodal region. Our study suggests that early-stage HPSCC with a PS apex extension might require intensive locoregional treatment for improving LRC; for example, adjuvant RT after surgery based on risk factors. Thus it can be presumed that elective neck treatment for early-stage HPSCC might have a role in regional control.

\section{Conflict of Interest}

No potential conflict of interest relevant to this article was reported.

\section{References}

1. Spector JG, Sessions DG, Haughey BH, et al. Delayed regional metastases, distant metastases, and second primary malignancies in squamous cell carcinomas of the larynx and hypopharynx. Laryngoscope 2001;111:1079-87.

2. Carpenter RJ 3rd, DeSanto LW, Devine KD, Taylor WF. Cancer of the hypopharynx: analysis of treatment and results in 162 patients. Arch Otolaryngol 1976;102:716-21.

3. Krstevska V. Early stage squamous cell carcinoma of the pyriform sinus: a review of treatment options. Indian J Cancer 2012;49:236-44.

4. National Comprehensive Cancer Network. Clinical guidelines in oncology-head and neck cancer-v. 1 [Internet]. Fort Washington, PA: National Comprehensive Cancer Network c2016 [cited 2016 Sep 6]. Available from: http://www.nccn. org/professionals/physician_gls/f_guidelines.asp.

5. Makeieff M, Mercante G, Jouzdani E, Garrel R, Crampette L, Guerrier B. Supraglottic hemipharyngolaryngectomy for the treatment of T1 and T2 carcinomas of laryngeal margin and piriform sinus. Head Neck 2004;26:701-5.

6. Plouin-Gaudon I, Lengele B, Desuter G, et al. Conservation laryngeal surgery for selected pyriform sinus cancer. Eur $J$ Surg Oncol 2004;30:1123-30.

7. Kuo CL, Lee $T L$, Chu PY. Conservation surgery for hypopharyngeal cancer: changing paradigm from open to endoscopic. Acta Otolaryngol 2013;133:1096-103.

8. Nakamura K, Shioyama Y, Kawashima M, et al. Multiinstitutional analysis of early squamous cell carcinoma of the hypopharynx treated with radical radiotherapy. Int J Radiat Oncol Biol Phys 2006;65:1045-50.

9. Mendenhall WM, Parsons JT, Stringer SP, Cassisi NJ, Million RR. Radiotherapy alone or combined with neck dissection for T1-T2 carcinoma of the pyriform sinus: an alternative 
to conservation surgery. Int J Radiat Oncol Biol Phys 1993;27:1017-27.

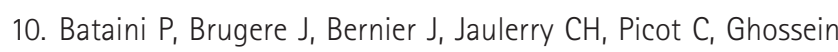
$N A$. Results of radical radiotherapeutic treatment of carcinoma of the pyriform sinus: experience of the Institut Curie. Int J Radiat Oncol Biol Phys 1982;8:1277-86.

11. Blanchard $P$, Tao $Y$, Veresezan 0 , et al. Definitive radiotherapy for squamous cell carcinoma of the pyriform sinus. Radiother Oncol 2012;105:232-7.

12. Sato $K$, Kubota $A$, Furukawa $M$, et al. Definitive radiotherapy for early-stage hypopharyngeal squamous cell carcinoma. Eur Arch Otorhinolaryngol 2015;272:2001-6.

13. Rabbani A, Amdur RJ, Mancuso AA, et al. Definitive radiotherapy for T1-T2 squamous cell carcinoma of pyriform sinus. Int J Radiat Oncol Biol Phys 2008;72:351-5.

14. Pameijer FA, Mancuso AA, Mendenhall WM, et al. Evaluation of pretreatment computed tomography as a predictor of local control in T1/T2 pyriform sinus carcinoma treated with definitive radiotherapy. Head Neck 1998;20:159-68.

15. Amdur RJ, Mendenhall WM, Stringer SP, Villaret DB, Cassisi NJ. Organ preservation with radiotherapy for T1-T2 carcinoma of the pyriform sinus. Head Neck 2001;23:353-62.

16. Buckley JG, MacLennan K. Cervical node metastases in laryngeal and hypopharyngeal cancer: a prospective analysis of prevalence and distribution. Head Neck 2000;22:380-5.

17. Koo BS, Lim YC, Lee JS, Kim YH, Kim SH, Choi EC. Management of contralateral NO neck in pyriform sinus carcinoma. Laryngoscope 2006;116:1268-72.

18. Ferlito $A$, Rinaldo $A$, Silver $C E$, et al. Elective and therapeutic selective neck dissection. Oral Oncol 2006;42:14-25.

19. Genden EM, Ferlito A, Bradley PJ, Rinaldo A, Scully C. Neck disease and distant metastases. Oral Oncol 2003;39:207-12.

20. Yoshimura R, Kagami $Y$, Ito $Y$, et al. Outcomes in patients with early-stage hypopharyngeal cancer treated with radiotherapy.
Int J Radiat Oncol Biol Phys 2010;77:1017-23.

21. Marta GN, Silva $V$, de Andrade Carvalho $H$, et al. Intensitymodulated radiation therapy for head and neck cancer: systematic review and meta-analysis. Radiother Oncol 2014;110:9-15.

22. Gupta T, Agarwal J, Jain S, et al. Three-dimensional conformal radiotherapy (3D-CRT) versus intensity modulated radiation therapy (IMRT) in squamous cell carcinoma of the head and neck: a randomized controlled trial. Radiother Oncol 2012;104:343-8.

23. Ferlito $A$, Silver $C E$, Rinaldo A. Neck dissection: present and future? Eur Arch Otorhinolaryngol 2008;265:621-6.

24. Schmitz S, Machiels JP, Weynand B, Gregoire V, Hamoir $M$. Results of selective neck dissection in the primary management of head and neck squamous cell carcinoma. Eur Arch Otorhinolaryngol 2009;266:437-43.

25. Johnson JT, Bacon GW, Myers EN, Wagner RL. Medial vs lateral wall pyriform sinus carcinoma: implications for management of regional lymphatics. Head Neck 1994;16:401-5.

26. Amar A, Dedivitis RA, Rapoport A, Quarteiro AL. Indication of elective contralateral neck dissection in squamous cell carcinoma of the hypopharynx. Braz J Otorhinolaryngol 2009;75:493-6.

27. Kowalski LP, Santos CR, Magrin J, Scopel A. Factors influencing contralateral metastasis and prognosis from pyriform sinus carcinoma. Am J Surg 1995;170:440-5.

28. Cooper JS, Zhang Q, Pajak TF, et al. Long-term follow-up of the RTOG 9501/intergroup phase III trial: postoperative concurrent radiation therapy and chemotherapy in high-risk squamous cell carcinoma of the head and neck. Int J Radiat Oncol Biol Phys 2012;84:1198-205.

29. Takes RP, Strojan $P$, Silver $C E$, et al. Current trends in initial management of hypopharyngeal cancer: the declining use of open surgery. Head Neck 2012;34:270-81. 